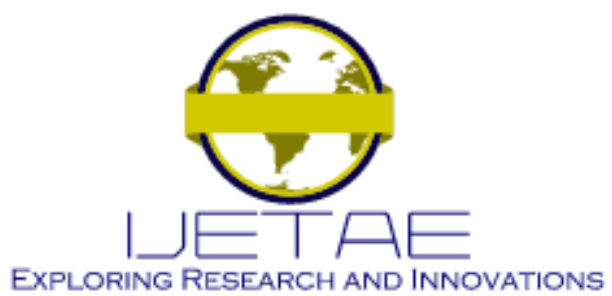

International Journal of Emerging Technology and Advanced Engineering

Website: www.ijetae.com (E-ISSN 2250-2459, Scopus Indexed, ISO 9001:2008 Certified Journal, Volume 11, Issue 07, July 2021)

Manuscript Received: 26 April 2021, Received in Revised form: 30 June 2021, Accepted: 05 July 2021

DOI: 10.46338/ijetae0721_02

\title{
A Novel Quantum Communication Protocol and its Simulation in IBM Quantum Simulator
}

\author{
Hai T. Nguyen ${ }^{1}$, Giao N. Pham ${ }^{2}$, Binh A. Nguyen ${ }^{3}$, Tung V. Nguyen ${ }^{3}$, Manh Hoang ${ }^{3}$ \\ ${ }^{1}$ Hutech Institute of Engineering, Ho Chi Minh City University of Technology (HUTECH), Vietnam \\ ${ }^{2}$ Dept. of Computing Fundamentals, FPT University, Hanoi, Vietnam \\ ${ }^{3}$ ICT Department, FPT University, Hanoi, Vietnam
}

\begin{abstract}
The paper gives the demonstrates of quantum super dense protocol and its related. Firstly, Qiskit's simulator is used to set-up and run the proposed quantum circuit, and then IBM quantum computer will be used to run the circuit for evaluation. In addition, we also introduce the two types of attacks which are possible on communication system and its simulation on IBM quantum computer via using quantum super dense protocol. The main findings are discussed in the wider setting of quantum cyber-security, where ways of hacking including the role of insider threats are discussed.

Keywords - Quantum information, Quantum communication protocol, Quantum super dense coding, Quantum simulator, Quantum IBM Computer, Qiskit's simulator.
\end{abstract}

\section{INTRODUCTION}

The next revolution in information and communications technologies (ICTs) will come from a development of a Quantum Internet (QI), integrating quantum communications and quantum artificial intelligence (QAI), with companies and states cooperating and competing on a strategic advantage over the development of quantum technologies: the race for quantum supremacy [1]. The development of an artificially intelligent quantum world wide web may produce a new civilizational jump, greater than the Fourth Industrial Revolution. In terms of foreseeable quantum technologies integration scenario, the most probable one is an accelerated integration of quantum computation and quantum communications in a hybrid quantum/classical infrastructure, until a full transition is achieved, as the research on nanotechnology and biotechnology is synergized with the research on quantum technologies, cloud and edge computing, quantum biology and open quantum systems, from the moment in which the full transition is achieved, quantum technologies will become the disruptive driver of the above-mentioned civilizational transition [2].
This future is exponentially near, we are already using nanotechnology and biotechnological hybridization to expand computing, companies and states are already in a race for the development of quantum technologies, a race where China has officially established landmarks in regards to quantum communications, but, at the black budget advanced research level, we cannot know how far ahead countries like the USA or Russia currently are, an arms race involves some secrecy, and there is a certain tradition to assure a strategic advantage first at a classified level before official disclosure. Also, at the level of quantum biology, the occurrence of quantum dynamics in biological systems may offer a way for the development of new directions into quantum technologies and open quantum computation.

From a computer science standpoint, a reflection on cyber-security within the context of quantum computation and communications is, thus, becoming urgent [5]. Under this context, if a quantum communications protocol has been hacked with installed malware this may lead to a corruption of the interaction with the networked quantum computational, communicational infrastructure. From a hacker's standpoint, this is not about eavesdropping on a quantum communications channel, as stated above, but rather about installing malware that can disrupt quantum communications. In the current article, we deal with this framework, such that the hacking is assumed to be done by replacement of code for automated gate specification in automated communications protocols. We analyse different attack patterns to a well-known quantum communications protocol: quantum superdense coding.

The work is divided into two sections. In section 2, we review the superdense coding protocol, its simulation in Qiskit's simulator and IBM quantum computers [3, 4]. In section 3, we present the bijection attacks and the scrambling attacks, simulating these attacks on IBM's quantum computers. 


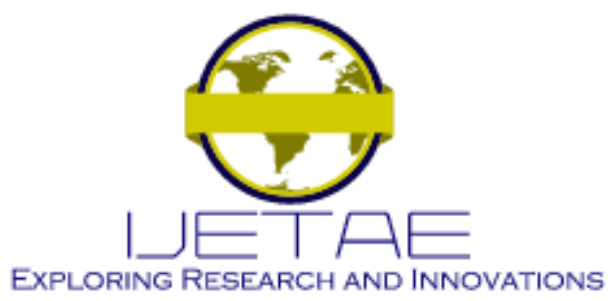

International Journal of Emerging Technology and Advanced Engineering

Website: www.ijetae.com (E-ISSN 2250-2459, Scopus Indexed, ISO 9001:2008 Certified Journal, Volume 11, Issue 07, July 2021)

\section{QUANTUM SUPER DENSE CODING}

Quantum information processing is the interdisciplinary researches of the processing techniques on basic information units which are working under the role of quantum mechanism. Then, it opens up the trend of using new material such as photons, light, trap-ions, ... for communication, network, securities protocols, for examples, some special techniques such as quantum teleportation, quantum superdense coding...

In this section, we will consider about quantum communication technologies via two most important protocols: quantum teleportation and quantum superdense coding. In overall, we can understand that quantum teleportation is the protocol for transmission of unit basic of quantum information system (qubits transmission) and quantum superdense coding is the transmission classical bits via quantum system by embedded or modulated classical bits into qubits.

TABLE I QUANTUM TELEPORTATION AND QUANTUM SUPER DENSE

\begin{tabular}{|l|l|}
\hline \multicolumn{1}{|c|}{ Superdense Coding } & \multicolumn{1}{c|}{ Teleportation } \\
\hline $\begin{array}{l}\text { Transmit classical bits } \\
\text { via quantum system. }\end{array}$ & $\begin{array}{l}\text { Transmit qubit between two } \\
\text { entities. }\end{array}$ \\
\hline
\end{tabular}

Process of quantum super dense coding is explained in diagram of figure 1 . The purpose of communication protocol in figure 1 is to exchange the information (bits) between two stations named Alisa and Babosa with medium is a third party named Charlie.

At first, Charlie will prepare the two qubits system which is the Bell entanglement state. After the Bell state is established, Charlie will send first qubit to Alisa and second qubit to Babosa.

Secondly, Alisa will apply two information bits into the qubit system shared by Charlie. Depending on the values of two bits she wants to send to Babosa, she will apply the different way of encoding.

Thirdly, Babosa will apply only decoding modules of CNOT and Hadamard gate; then, he will perform the measurement to know exactly bits sent by Alisa. The full equations and case of three steps are given at Figure 2.

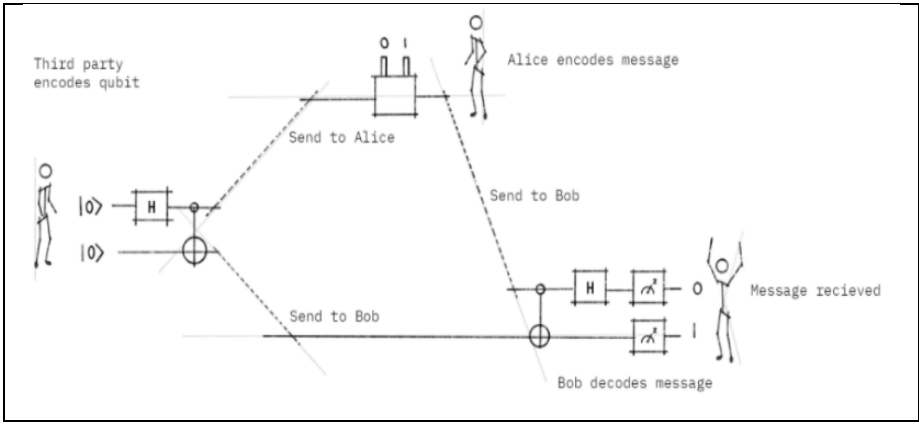

FIGURE I: THE STEPS OF QUANTUM SUPER DENSE

\section{\#Step 1:}

$$
\begin{aligned}
& |0+\rangle=\frac{1}{\sqrt{2}}(|00\rangle+|01\rangle) \\
& \operatorname{CNOT} \frac{1}{\sqrt{2}}(|00\rangle+|01\rangle)=\frac{1}{\sqrt{2}}(|00\rangle+|11\rangle)
\end{aligned}
$$

\begin{tabular}{ccc}
\hline $\begin{array}{l}\text { \#Step 2: } \\
\text { Intended Message }\end{array}$ & Applied Gate & Resulting State $(\cdot \sqrt{2})$ \\
\hline 00 & $I$ & $|00\rangle+|11\rangle$ \\
10 & $X$ & $|01\rangle+|10\rangle$ \\
01 & $Z$ & $|00\rangle-|11\rangle$ \\
11 & $Z X$ & $-|01\rangle+|10\rangle$
\end{tabular}

Step 3:

Bob Receives: After CNOT-gate: After H-gate:
$|00\rangle+|11\rangle$
$|00\rangle+|01\rangle$
$|00\rangle$
$|01\rangle+|10\rangle$
$|11\rangle+|10\rangle$
$|10\rangle$
$|00\rangle-|11\rangle$
$|00\rangle-|01\rangle$
$|01\rangle$
$-|01\rangle+|10\rangle$
$-|11\rangle+|10\rangle$
$-|11\rangle$

FIGURE II. DETAIL EQUATIONS AND CASED FOR THREE STEPS OF QUANTUM SUPER DENSE CODING PROCESS 


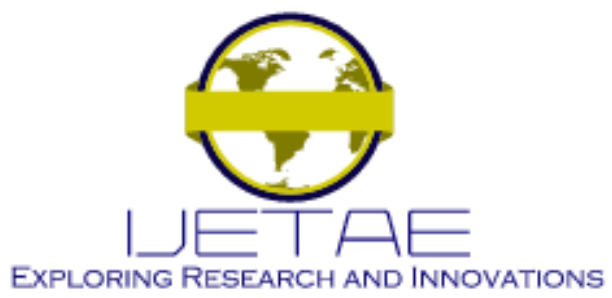

International Journal of Emerging Technology and Advanced Engineering

Website: www.ijetae.com (E-ISSN 2250-2459, Scopus Indexed, ISO 9001:2008 Certified Journal, Volume 11, Issue 07, July 2021)

Secondly, Alisa will apply two information bits into the qubit system shared by Charlie. Depending on the values of two bits she wants to send to Babosa, she will apply the different way of encoding.

Thirdly, Babosa will apply only decoding modules of CNOT and Hadamard gate; then, he will perform the measurement to know exactly bits sent by Alisa. The full equations and case of three steps are given at Figure 2.

\section{Simulation Of Quantum Super Dense Coding Via IBM QUANTUM COMPUTER}

\section{A. Super dense coding via Qiskit simulator}

In this section, we will use an open source SDK named Qiskit for simulating our proposed quantum algorithm at the low level. Qiskit SDK is built up by pulses, circuits, and sub-modules. In addition, many well-researches in quantum module circuits have been packed and ready to use in the Qiskit library. As the consequence, a lot of prototypes have been evaluated via Qiskit. The following programs are the evaluation process for our proposed superdense coding algorithm via Qiskit platform.

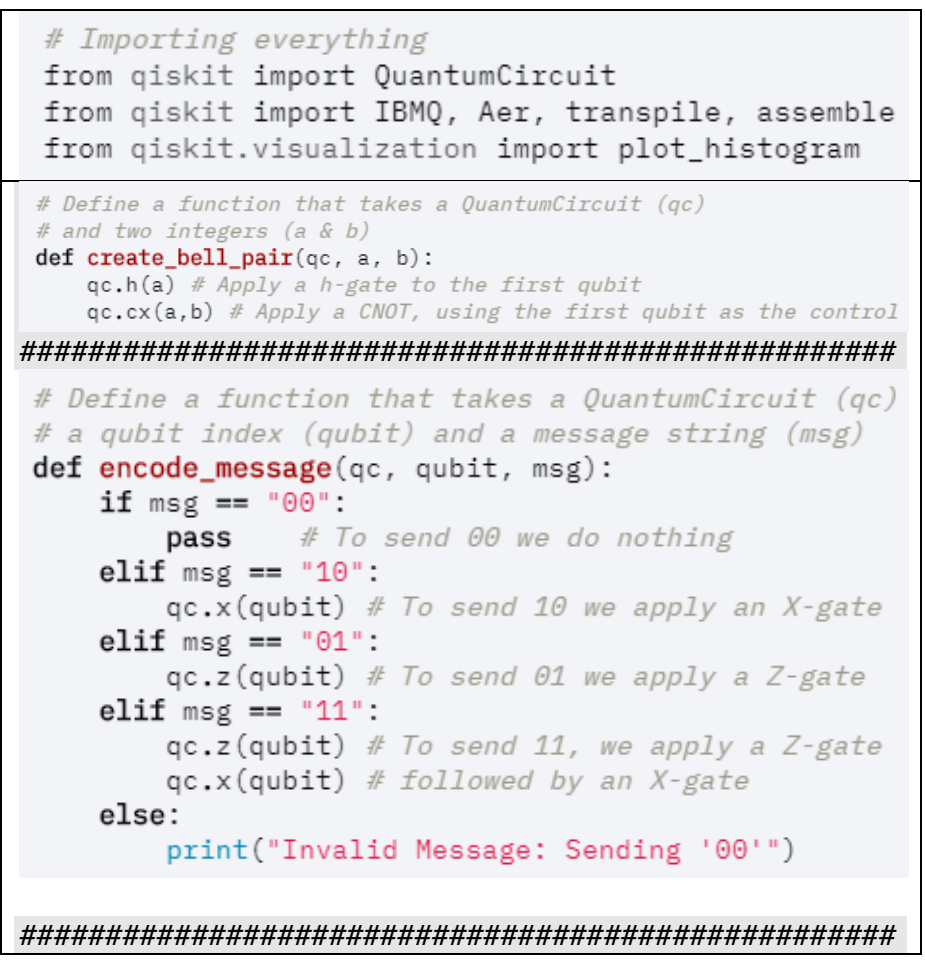

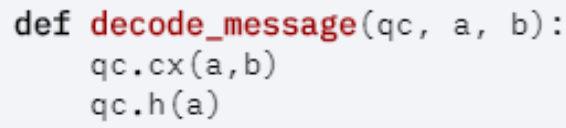

\# Create the quantum circuit with 2 qubits qc = QuantumCircuit (2)

\section{\#\#\#\#\#\#\#\#\#\#\#\#\#\#\#\#\#\#\#\#\#\#\#\#\#\#\#\#\#\#\#\#\#\#\#\#\#\#\#\#\#\#}

\# First, Charlie creates the entangled pair between Alice and Bob create_bell_pair(qc, 0,1$)$

qc.barrier() \# This adds a barrier to our circuit. A barrier

\# separates the gates in our diagram and makes it

\# clear which part of the circuit is which

\#\#\#\#\#\#\#\#\#\#\#\#\#\#\#\#\#\#\#\#\#\#\#\#\#\#\#\#\#\#\#\#\#\#\#\#\#\#\#\#\#\#

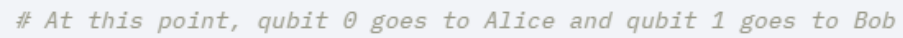

\# Next, Alice encodes her message onto qubit 0 . In this case,

\# we want to send the message '10'. You can try changing this

\#⿰ value and see how it affects the circuit

message $=$ "10"

encode_message ( $q c, 0$, message)

qc.barrier()

\# Alice then sends her qubit to Bob.

\# After recieving qubit $\Theta$, Bob applies the recovery protocol: decode_message $(q c, 0,1)$

\# Finally, Bob measures his qubits to read Alice's message qc.measure_all()

\# Draw our output

qc.draw ()

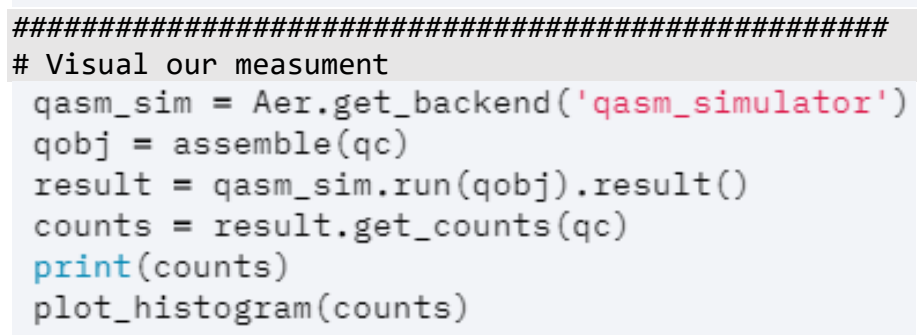

\section{FIGURE III. THE STEPS OF QUANTUM SUPER DENSE CODING} IN QUISKIT SIMULATOR

The quantum circuit as the example is given as following synthesis. 


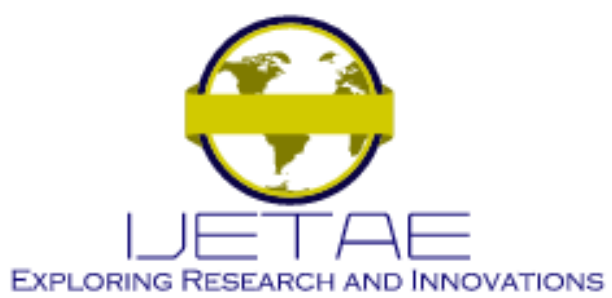

International Journal of Emerging Technology and Advanced Engineering

Website: www.ijetae.com (E-ISSN 2250-2459, Scopus Indexed, ISO 9001:2008 Certified Journal, Volume 11, Issue 07, July 2021)

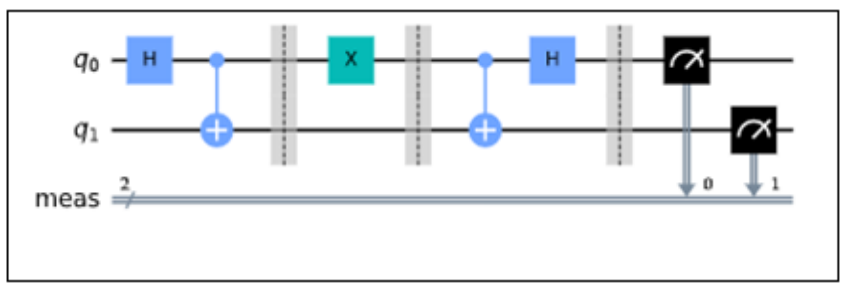

The measurement result is given as following.

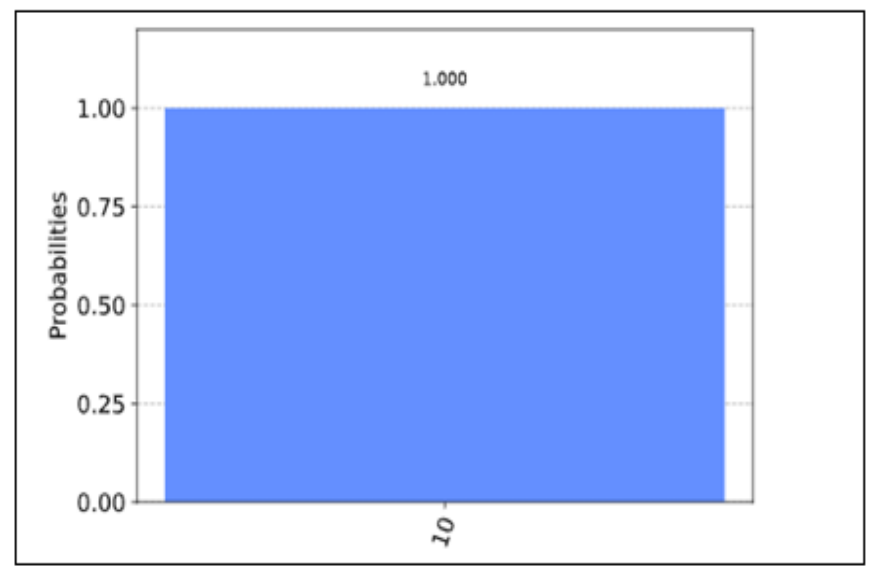

B. IBM quantum computer

The circuit, for the above communication dynamics, to be simulated via a computer based simulator which is working under the quantum mechanism, has the following structure:

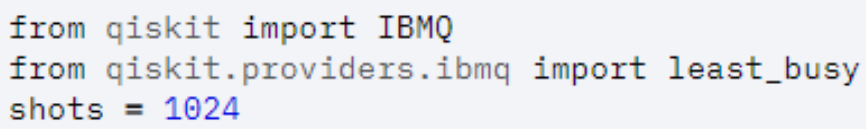

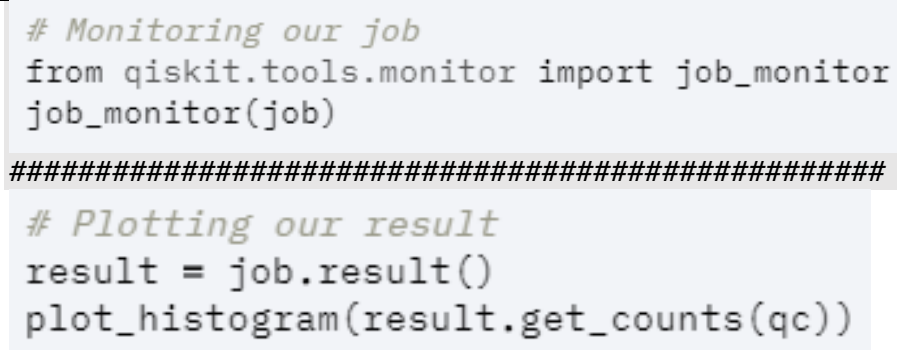

As can be seen from the figures, there are some errors from the results of other three states and the results in quantum computer simulator. The different between them are from the de-coherence in quantum gates.

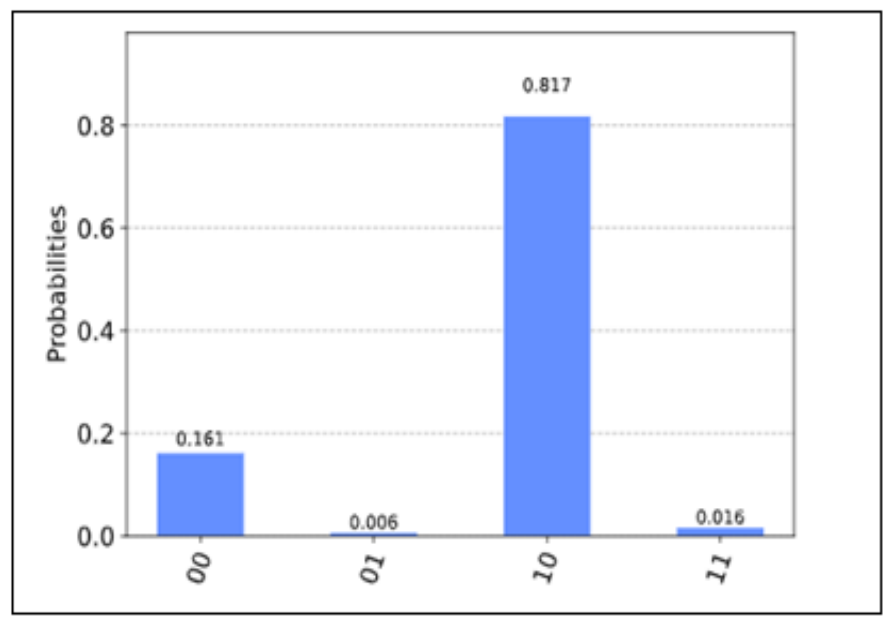

\section{CONSLUSION}

Quantum information processing has promised and opened the new role on the next information generation due to the advantages on securities, information storage, and information processing. The paper has discussed the major step on design of quantum information algorithm based on the Qiskit emulator and IBM quantum computer. It is a good reference for students and researchers on the field of research.

\section{Acknowledgement}

This work is supported by FPT University, Hanoi, Vietnam; and Hutech Institute of Engineering, Ho Chi Minh City University of Technology (HUTECH), Vietnam 


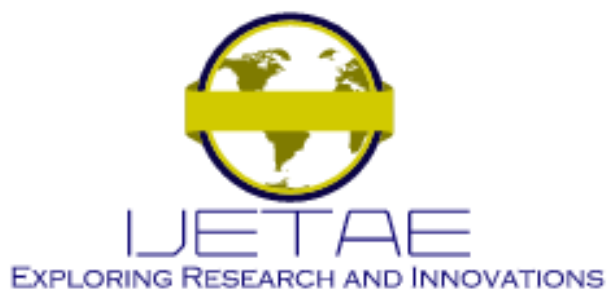

International Journal of Emerging Technology and Advanced Engineering Website: www.ijetae.com (E-ISSN 2250-2459, Scopus Indexed, ISO 9001:2008 Certified Journal, Volume 11, Issue 07, July 2021)

\section{REFERENCES}

[1] Binh, A.N. et. al. A Novel Framework for Simulation of Quantum Information System. International Journal of Advanced Trends in Computer Science and Engineering. 9(2), 1752-1756, 2020.

[2] Nguyen, D. M. et. al. New Constructions of Quantum Stabilizer Codes Based on Difference Sets. Symmetry 10(11), 655. 2018.
[3] https://qiskit.org/

[4] https://qiskit.org/textbook/ch-algorithms/superdense-coding.html

[5] Daniel J. F. et. al. Development of an Automated MATLAB-Based Platform for the Analysis of Massive EEG Datasets. International Journal of Emerging Technology and Advanced Engineering. 10(11), 2020 